\title{
Visual cliff behavior of mice as a function of genetic differences in eye characteristics'
}

\author{
RICHARD FRANK, FLUSHING, NEW YORK \\ JOHN KENTON2, FAIRFIELD UNIVERSITY
}

The effects of type of breeding on visual cliff avoidance is evaluated using three groups of mice with different genetically determined eye characteristics-pigmented (P), nonpigmented (NP) and rod-deficient (RD)-tested on 2 occasions. The results show significant group differences on latency and descents. P and NP groups show high degree of cliff avoidance and NP significantly less descents than P. Strain differences were evident on latency and total descents for RD and on bolus count for NP. Latency decreased significantly from first to second test. Inter-and intraspecies differences are emphasized and genetic analysis of determinants of cliff avoidance suggested.

Cliff-avoidance behavior is known to be a widespread response exhibited by many different organisms (Gibson \& Walk, 1960; Routtenberg \& Glickman, 1964a, 1964b). Also intraspecies differences have been established (Routtenberg \& Glickman, 1964a). Yet as the latter authors point out (1964b): "It cannot be stated with certainty, however, whether a type of breeding process has produced animals with specialized sensitivity to height."

Perhaps more is known of the genetic background of the mouse than of any other animal suitable for visual cliff experiments. Since many inbred strains have been maintained for over 20 generations by brother-sister matings, the mouse is particularly well suited for evaluating the effects of selective breeding. This study examines cliff avoidance on two occasions of strains differing in genetically determined eye characteristics (pigmented, non-pigmented and rod-deficient eyes). Method

Three groups of Ss were used: mice with pigmented eye (P), non-pigmented eye (NP) and rod-deficient eye (RD). The $P$ group consisted of one hybrid and two inbred strains (C57 BL/6J, LP/J, and B6D2F 1 ) with normal retina and pigmented eyes. The parent strains of B6D2 $F_{1}$ hybrid are C57BL/6Jo and DBA/2Jo. The NP group consisted of three inbred strains $(129 / \mathrm{J}$, $\mathrm{BALB} / \mathrm{CJ}$, and $\mathrm{RF} / \mathrm{J}$ ) with normal retina and nonpigmented eyes. The RD group consisted of three inbred strains (SJL/J, C3H/HeJ, and SWR/J) with hereditary retinal degeneration of the rod layer. Six Ss of each strain were included, giving a total of 18 Ss per group. All Ss were male, naive, and 40-49 days of age at time of testing, except Ss of the LP/J strain, which were 42-55 days old. In home cages the Ss were on ad libitum food and water.

The basic design of the visual cliff followed that described by Routtenberg \& Glickman (1964). It con- sisted of a wooden frame $16.5 \mathrm{in}$. wide, $21.5 \mathrm{in}$. long and 31 in. in depth, into which a piece of "Plexiglas" 16.5 in. $x 20$ in. had been placed, 24 in. above the floor in a horizontal position. A red, pink, and white checkerboard pattern on oilcloth of $7 / 16$ in. squares (alternate rows white and pink squares and red and pink squares) was placed directly under one half of the glass (shallow side) and on the floor under the other half (deep side). The centerboard, 16.5 in. $\times 3.5$ in. $\times 1.5$ in. covered with the same material, was placed across the center of the glass, dividing the surface in half. The "Plexiglas" platform and centerboard were illuminated by a floodlight directed downward and along the centerboard. Another floodlight was directed across the floor of the deep side. The mice were contained by walls of cloth tacked onto the frame.

Each $S$ was placed on the centerboard (facing away from E) and observed for a 5-min. period. After the testing of each $\mathrm{S}$, the centerboard and glass were cleaned with a damp cloth. The following measures were recorded: side and latency of first descent, total time on shallow and deep, total number of descents to shallow and deep, and count of boluses. The E observed from a position in line with the centerboard. The procedure was carried out twice for every $S$, with a two day interval between test and retest.

(An apparatus control was run to determine any possible differences in the two sides other than the cliff effect. With the pattern directly beneath the glass on both sides (shallow position), a group of 84 animals, independent of the Ss described above and composed of six strains, was tested. Of the 65 Ss descending from the centerboard, 35 went left and 30 right.)

Results

The main analyses show that groups differ significantly on several measures, and suggest differential reactions attributable to strain differences.

The number of Ss in each group descending in the $5-$ min. session were 16,8 , and 11 for $P, N P$, and RD respectively $\left(x^{2}=7.96, \mathrm{df}=2, \mathrm{p}<.02\right)$. The NP mice descended significantly less than the $\mathrm{P}$ mice $\left(x^{2}=\right.$ $8.00, \mathrm{df}=1, \mathrm{p}<.01$ ). Initial choice was clearly for the shallow side: none of the P or NP group descended to the deep side compared with four deep choices for the RD group ( $x^{2}=9.87, \mathrm{df}=2, \mathrm{p}<.01$ ). Even during the full session only one $S$ in each of the $P$ and NP groups descended to the deep side compared with 9 of the RD group $\left(X^{2}=18.98, \mathrm{df}=2, \mathrm{p}<.001\right)$. A one-way analysis of variance yielded significant differences for latency ( 5 min. $\max . ; F=9.073, \mathrm{df}=2 / 51, \mathrm{p}<.001$ ) and total number 
of descents ( $\mathrm{F}=5.287, \mathrm{df}=2 / 51, \mathrm{p}<.01$ ). The difference between the mean time spent on the shallow versus the deep side for the RD group-the only group to descend ennsistently to both sides-was not significant (M shallow $=107.40$ sec., $M$ deep $=119.67 \mathrm{sec}$.)

A one-way analysis of variance was applied to within group differences for strains. The F-ratio for latency of descent was significant beyond the .001 level for the RD group ( $F=18.15, d f=2 / 15)$, the $S J L$ showing shorter latency than the SWR and $\mathrm{C} 3 \mathrm{H}$. The only significant difference in total descents was again the $\mathrm{RD}$ group $(\mathrm{F}=56.618, \mathrm{df}=2 / 15, \mathrm{p}<.001)$ and attributable to the SJL strain with the highest number of descents. For bolus counts the analysis was significant for the NP group $(\mathrm{F}=5.482, \mathrm{df}=2 / 15, \mathrm{p}<.05)$ with the BALB strain showing least counts.

On the second test the differences among groups comparing descenders and non-descenders was not significant (Descenders: $P=16, N P=10, R D=12$ ). However, on the initial choice the strong avoidance of the deep side of the P and NP groups was still evident, with no deep choices compared to 5 choices for the RD group $\left(x^{2}=12.48, \mathrm{df}=2, \mathrm{p}<.01\right)$. During the course of the second test the proportions of Ss going to the deep side were 5 of 16,2 of 10 , and 12 of 12 for the P, NP, and $R D$ groups respectively $\left(x^{2}=17.85, \mathrm{df}=2, \mathrm{p}<.001\right)$.

Latency was the only measure to show a significant shift from the first to the second test. Application of a two-way analysis of variance with one-way correlation yielded significant $F$-ratios for the main effects of session, decrease in latency $(\mathrm{F}=8.307, \mathrm{df}=1 / 51, \mathrm{p}<$ .01 ), and groups $(\mathrm{F}=7.642, \mathrm{df}=2 / 51, \mathrm{p}<.01)$. The interaction of latency and groups was not significant.

\section{Discussion}

In general mice $\checkmark$ various strains with normal retinas and pigmented and non-pigmented eyes show visual cliff avoidance similar to other organisms and stable responses, except latency of initial descent, over two test sessions.

One possible reason for the lower number of descents in the non-pigmented eye group is that a non-pigmented eye provides less discriminatory ability than a pigmented one (Lashley, 1930). However, this does not appear to be a sufficient explanation since those Ss to descend clearly manifested cliff avoidance. Taking bolus counts as a measure of emotionality the NP group did not differ significantly from the $P$ and $R D$ groups. A remaining possibility is that the mice in the NP group were simply slow to respond. Some support for this interpretation may be found in the data for the appa- ratus control group: only 7 of 12 of strain $129 / \mathrm{J}$ descended even when both sides were in the shallow position.

Evidence of a defect in visual discriminatory ability in strains $\mathrm{SJL} / \mathrm{J}, \mathrm{SWR} / \mathrm{J}$ and $\mathrm{C} 3 \mathrm{H} / \mathrm{HeJ}$ is seen in the lack of significant differences in responses to the shallow and deep sides. (Incidentally, this is additional evidence for the lack of difference between the two sides, apart from the visual cliff.) The results are consistent with the finding of Wimer \& Weller (1964) of an inability of these strains to learn a brightness discrimination. However, in the light of the differences between the $R D$ strains, it seems that accommodation to visual deficit is different for different strains.

In contrast with other studies (Routtenberg \& Glickman, 1964a, 1964b) the mice in this study show greater cliff avoidance than other rodents including spiny mice, and no difference between pigmented and non-pigmented eye. Further tests showed this discrepancy not to be a function of size of square-a difference between the two studies and the present one. (The 16 Ss of the $\mathbf{P}$ group which descended were divided into two groups, matched for total descents and strain, and tested without a centerboard. Time on deep and shallow was recorded, using the original square for one group and a $2-1 / 4$ in. square for the other, and mean percentages of time on deep were 28.00 and 21.00 respectively, a non-significant difference.) Apparently, the differences are inter- and intraspecies differences.

The mouse appears to be well suited for further genetic and behavioral analysis of visual cliff avoidance.

\section{References}

Gibson, E., \& Walk, R. D. The "visual cliff." Scient. American, $1960,202,64-71$

Lashley, K. S. The Mechanism of vision. III. The comparative visual acuity of pigmented and albino rats. $J$. genet. Psychol. $1930,37,481-484$.

Routtenberg, A., \& Glickman, S. E. Visual cliff behavior in albino and hooded rats. J. comp. physiol. Psychol., 1964a, 58, 140-142.

Routtenberg, A., \& Glickman, S. E. Visual cliff behavior in undomesticated rodents, land and aquatic turtles, and cats (panthera). J. comp. physiol. Psychol., 1964b, 58, 143-146.

Wimer, R., \& Weller, S. Evaluation of a visual discrimination task for the analysis of the genetics of mouse behavior. Percept. mot. Skills, 1965, 20, 203-208.

\section{Notes}

1. This investigation was supported by grants MH-1775 and NSFGE 7397. The authors wish to acknowledge the advice and assistance of Dr. R. E. Wimer, and the constructive commentary of Dr. Robert Plutchik, which led to the revised method of testing described in the discussion.

2. Now at Department of Psychology, University of Illinois, 907 South Sixth St., Champaign, Illinois. 